\title{
Convection laminaire et convection turbulente
}

\section{Laminar and turbulent convection}

\author{
PAR G. RIBAUD \\ MEMBRE DE L'INSTITUT, \\ PROFESSEUR HONORAIRE A IAA SORBONNE
}

\begin{abstract}
L'étude de la convection de la chaleur nécessite l'étude préalable des lois d'écoulement dni fluide. Deux types d'ecoulement sont à envisager dans chaque cas concret, un éconlement laminaire dans lequel les filets gazeux s'écoulent parallèlement sans se mélanger et un écoulement turbulent dans lequel, en chaque point, à la vitesse d'ensemble se superposen! des fluctuations aléatoires de vitesse à l'échelle macroscopique.

Il est tonjours possible d'écrire l'équation de transmission de la chaleur au sein du fluide, jusqu'à la paroi, chaque fois gue la loi d'écoulement est connuc. Mais. en fait, la résolution de cette équation n'est possible que dans des cas simples physiquement définis : convection le long d'un plan ou d'un obstacle profilé, convection dans un conduit circulaire en régime dynamique établi. L'auteur donne les formules simples relatives da ces cas concrets.

En revanche, chaque fois que le régime d'scoulement n'est pas établi, les lois de vitesse très compliquées interdisent toute résolution mathématique rigonreuse de l'équation de la chaleur. On en est alors réduit à des simplifications assez grossières fixant les ordres de grandeur des échanges thermiques.

Le problème des obstacles non profilés est également abordé; ici encore le calcul est inoperant, faute de connritre les lois du sillage en aval de l'obstacle; l'expérience permet tontefois d'obtenir le graphique universel fixant les échanges thermiques.
\end{abstract}

\section{Mestames, Messieurs,}

La Socićté Hydrotechnique a bien voulu me demander de vous faire, en une demi-heure, un exposé sur le problème de la convection de la chaleur. Je dois avouer que le sujet est extrêmement vaste et qu'il me sera impossible' de l'aborder complètement. Ce que je voudrais, par contre, e'est vous donner les bases théoriques essentielles nécessaires aux techniciens ayant à

\begin{abstract}
A study of heat convection requires a preiminary study of the fluids laws of flow. Two types of flow must be expected in each all actual examples; laminar flow in which the gas stream-lines flow parallel lo each other without mixing, and turbulent flow at each point of which the random velocity fuctuations are superimposed at macroscopic scale upon the lump velocity.

The equation of heat transfer wilhin the fluid up to the bounding walls can be written when ever the flow characteristics are linown. Bul in actual fact this equation can only be soved in simple cases which are deflned physically such as: convection in a plane or on a streamlined obstacle or convection in a circular pipe with an established dynamic state. The author gives simple formulae connected with these actual examples.

On the other hand, whenever flow is not established, very complicated velocily laws make impossible any rigorous solution of the heat transfer equation. Rather sweeping simplifications which give the orders of magnitude of the thermic exchanges, then have to be made. The problem of nonstreamlined obstacles is also touched upon; for his once again calculations are of little use since the wake laws below the obstacle are unknown. Nevertheless the universal graph showing heal exchanges can be drawn from experience.
\end{abstract}

aborder les problèmes de transmission de chaleur dans le domaine de la convection.

Je tiens d'abord à rappeler les grandeurs fondamentales dont j'aurai à me servir au cours de cet exposé.

\section{Grandeurs dynamiques}

En premier lieu, la convection étant liée intimement à l'écoulement du fluide, il est néces- 
saire de définir d'abord Jes constantes dynamiques de ce fluide. En dehors de la vitesse $u$ en chaque point, il $y$ a lieu de considérer la masse spécifique $?$ et la viscosité $u$ du lluide, le rapport $\mu / p$ (viscosité cinématique) s'exprime par la notation v. Par ailleurs, la nature de l'écoulement (laminaire ou turbulent) sera régie par un nombre sans dimensions, appelé nombre de Reynolds, défini par la relation :

$$
\mathscr{R}=\frac{u l ?}{\mu}=\frac{u l}{v}
$$

dans laquelle la grandeur $l$ est une longueur caractéristique (parcours le long d'un plan, diamètre d'un conduit ou d'un obstacle, par exemple).

\section{Grandeurs thermiques}

En ce qui concerne les grandeurs calorifiques relatives au fluide, il $\mathrm{y}$ a lieu de considérer, d'une part la conductibilité thermique $\lambda$, d'autre part la chaleur spécifique $\mathrm{C}$.

Nous aurons en outre à considérer le nombre sans dimensions (nombre de Prandtl-Stanton) dont l'expression est :

$$
\mathscr{P}=\frac{\mathrm{C} \mu}{\lambda}
$$

nombre qui associe les propriétés thermiques et dynamiques $\mathrm{du}$ fluide.

\section{Coefficient de convection}

Enfin, en ce qui concerne le transfert de chaleur entre le fluide et une surface donnée, nous utiliserons le coefficient de convection $\alpha$ défini comme étant le rapport entre la quantité de chaleur $q$ gagnée ou perdue (par unité de temps, et par unité de surface), et l'écart de température entre fluide et surface.

Dans les théories de la convection on est amené à utiliser le nombre sans dimension $\mathcal{B}=\alpha l / \lambda$ (nombre de Biot-Nusselt dans Iequel $l$ est ici encore une longueur caractéristique choisie judicieusement dans chaque cas) ou encore lautre nombre sans dimension $\mathfrak{N}=\alpha / c ? u_{0}$ (nombre de Margoulis).

\section{Ecoulement laminaire et turbulent}

Dans un écoulement purement laminaire, les filets fluides ne se mélangent pas; les échanges de maticre d'une couche à la couche voisine s'effectuent uniquement grâce à l'agitation moléculaire. Il en résulte, pour divers gaz, que le nom- bre de Prandtl $C_{\mu} / \lambda$. est voisin de l'unité. Pour les liquides non métalliques il peut aller de quelques unités (eau, alcools...) à quelques centaines d'unités pour les liquides visqueux (glycérine, huiles); quant aux liquides métalliques (mercure, métaux ou alliages fondus), leur nombre de Prandtl est de l'ordre de 0,1 à 0,01 .

Dans un écoulement turbulent, les filets fluides ne conservent plus leur individualité mais se mélangent progressivement grâce à des tourbillons transportant des quantités macroscopiques de matière. Nous verrons plus loin dans quelles conditions s'amorce un tel écoulement turbulent.

\section{Transfert de chaleur le long d'un plan en régime laminaire}

Pour étudier ce problème, comme tous ceux qui suivront, il est indispensable de faire une étude préalable du phénomène d’écoulement. l'expérience monlre que, lorsqu'un fluide s'écoule le long d'un plan ox (lig. 1), il se forme

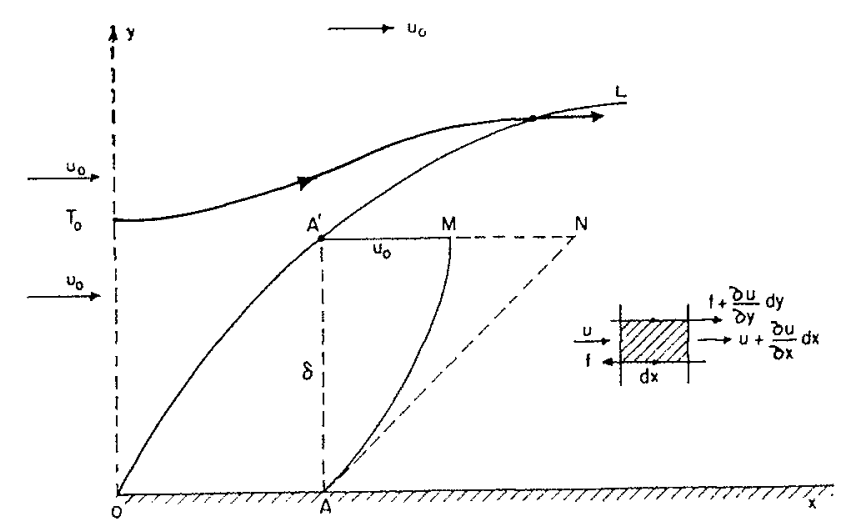

Fici. 1

le long de ce plan une couche limite OL dans laquelle la vitesse, nulle à la paroi, croît progressivement jusqu'à la valeur $u_{0}$ à la frontière de la couche limite.

La mise en équation du problème dynamique ne comporte aucune difficulté. En se limitant aux termes essentiels, si l'on considère le parallélépipède en hachures, on peut écrire que la perte de quantité de mouvement o $\boldsymbol{u}(\partial \boldsymbol{u} / \partial \boldsymbol{x})$. du fluide entre les plans $x$ et $x+d x$, est égale à la résultante des forces de viscosité agissant sur les deux laces $y$ et $y+d y$, résultante qui a poux valeur :

$$
\frac{\partial}{\partial y}\left(\text {, } \frac{\partial u}{\partial y}\right)==: \frac{\partial^{\prime} \prime \prime}{\partial y^{2}},
$$

de sorte que l'équation régissant l'écoulement s'erit :

$$
u \frac{\partial u}{\partial x}=\frac{\mu \cdot \partial^{2} u}{\rho} \frac{\partial y^{2}}{2}
$$


La résolution de cette équation, effectuée avec le changement de variable $z=y / \sqrt{x}$, conduit aux résultats suivants. L'épaisseur de la couche limite $\delta$ est donnée par la relation :

$$
\delta=6 \sqrt{\frac{v x}{u_{u}}}
$$

Quant à la distribution des vitesses dans l'épaisseur $\delta$, elle peut, en première appproximation, être représentée par la parabole AM d'équation :

$$
\frac{u}{u_{0}}=\frac{2 y}{\delta}-\frac{y^{2}}{\delta^{2}}
$$

Du point de vue thermique, prenons pour zero la température du plan et appelons $0_{0}$ la diffèrence de température : fluide à l'infini-plan. Pour le parallélépipède en hachures, nous pouvons écrise quc la quantité de chaleur entrainée par le fluide entre les plans $x$ et $x+d x[\circ \mathrm{C} u(\partial \mathrm{T} / \partial x)]$ est égale à la quantité de chaleur $\partial / \partial y[\lambda(\partial \mathrm{T} / \partial y)]$ accumulée entre $y$ et $y+d y$ par la conduction thermique, d'où l'équation :

$$
u \frac{\partial \mathrm{T}}{\partial x}=\frac{\lambda}{\mathrm{C}_{\rho}} \frac{\partial^{2} \mathrm{~T}}{\partial y^{2}}
$$

On remarque tout de suite la similitude entre les équations (1) et $(1 \mathrm{bis})$; les coefficients de leurs seconds membres sont entre eux dans le rapport $(\mathrm{C} ; / \lambda)=\mathscr{Q}$.

Pour les gaz dont le nombre de Prandtl est voisin de l'unité, il y a similitude des profils des températures et des vitesses, de sorte que la répartition des températures dans la couche limite peut s'écrire :

$$
\frac{\mathrm{T}}{\mathrm{T}_{0}}=\frac{2 y}{\delta}-\frac{y^{2}}{\delta^{2}}
$$

et la quantité de chaleur $q$ amenée à la paroi par unité de surface et unité de temps au point d'abscisse $x$, va s'exprimer par :

$$
q=\lambda\left(\frac{\partial \mathrm{T}}{\partial y}\right)_{y=0}=\frac{2 \lambda}{\delta} \mathrm{T}_{0}
$$

et le coefficient de convection $\alpha_{x}$ par :

$$
\alpha_{x}=\frac{\gamma}{T_{0}}=\frac{2 \lambda}{\delta}=\frac{\lambda}{3} \sqrt{\frac{u_{0}}{v x}}
$$

Le coefficient moyen entre 0 et $x$ a la valeur double. Si le nombre de Prandtl n'est pas égal à l'unité, on démontre aisément que la couche limite «thermique», analogue à la couche $d y-$ namique, a une épaisseur $\Delta$ reliée à $\delta$ par la relation :

$$
\Delta=\delta \mathscr{P}^{1 / 3}
$$

de sorte que le coefficient de convection s'écrit, dans le cass général :

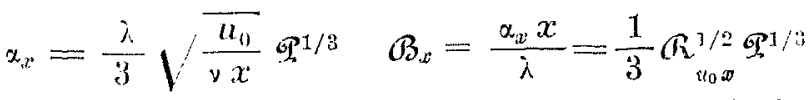

(4 bis)

Les formules précédentes ne sont valables que si l'écoulement reste laminaire. Si le parcours $l$ le long du plan est suffisant, il finit par s'amorcer un régime turbulent dont le schéma est donné par la figure 2. Nous reviendrons plus loin sur ce régime qui s'amorce pour une valeur de $u_{0} l / v$ voisine de $5.10^{5}$.

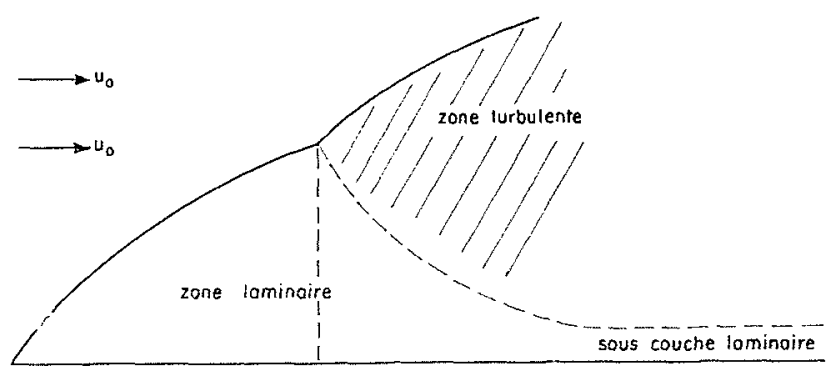

Fra. 2

\section{Convection laminaire dans un conduit circulaire}

Ici encore, il est nécessaire de bien préciser les phénomènes d'écoulement avant d'aborder le problème de la convection. Si un fluide pénètre dans un conduit avec la vitesse uniforme $u_{0}$, il s'amorce, dès l'entrée, une couche limite ơL (fig. 3) qui finit par emplir totalement le conduit.

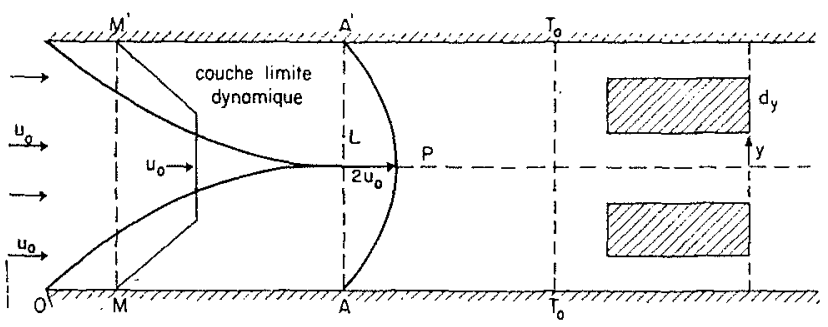

Fig. 3

Le parcours $\mathrm{OA}=l$ nécessaire pour l'établissement du régime est tel que $l / d=0,06 \cdot \mathcal{R} u_{0} d$, $d$ représentant le diamètre du conduit. Pour un nombre de Reynolds égal à 1.000 , la longueur $l$ nécessaire est égale à 60 fois le diamètre du conduit.

La vitesse sur l'axe, égale à $u_{0}$ à l'entrée, croît jusqu'à devenir égale à $2 n_{0}$; la surface des vitesses, en régime dynamique ćtabli, est un paraboloïde APA' dont la méridienne s'exprime par :

$$
\frac{u}{2 u_{0}}=1-\frac{y^{2}}{r^{2}}
$$


La valeur moyenne de la vitesse dans une section est évidemment égale à $u_{0}$.

Le calcul de l'échange par convection entre fluide et paroi n'a pu être effectué qu'à partir du moment où le régime dynamique est établi; envisageant ce cas, nous allons supposer que la paroi, initialement à la même température que le fluide, est portée brusquement à une température $\mathrm{T}_{0}$ inférieure à celle du fluide; il revient d'ailleurs au même de considérer la paroi à la température zéro et le fluide initialement à la température $T_{0}$.

La mise en équation du problème thermique ne comporte ici encore aucune difficulté. Si l'on considère le tore en hachures de la figure 3 , la quantité de chaleur enlevée par le fluide a pour valeur $\mathrm{C}$ ? $u(\partial \mathrm{T} / \partial x)$, elle doit être égale à celle laissée par la conduction $\lambda(\partial / \partial y)[2 \pi y(\partial \mathrm{T} / \partial y)]$. La résolution assez délicate de l'équation ainsi obtenue conduit aux résultats suivants (fig. 4).

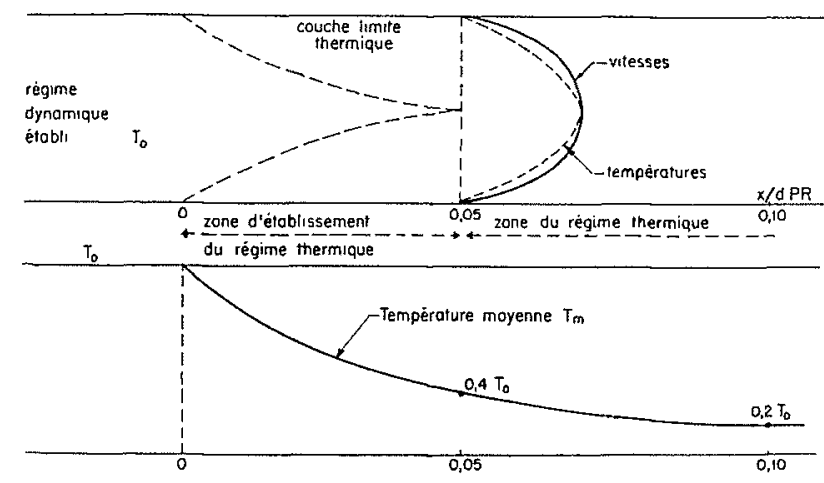

FIc. 4.

Le fluide conserve, sur l'axe, sa température initiale pendant un parcours suffisant pour que la couche limite thermique emplisse toute la section du conduit. Ce parcours $l^{\prime}$ est tel que :

$$
\frac{r}{d}=0,05 \Re \mathscr{T}
$$

La température moyenne $\mathrm{T}_{m} \mathrm{du}$ fluide, au bout de ce parcours, atteint la valeur $0,4 \mathrm{~T}_{0}$; après un parcours double, la valeur $0,2 \mathrm{~T}_{0}$; quand le régime thermique est établi, le coefficient de convection, rapporté à la température moyenne du fluide, prend l'expression simple:

$$
\alpha=3,66 \frac{\lambda}{d} \quad \text { ou } \quad \mathfrak{B}=\frac{\alpha d}{\lambda}=3,66
$$

ce qui revient, en fait, à dire que la quantité de chaleur cédée à la paroi ne dépend pas du diamètre du conduit, mais uniquement de la température moyenne du fluide.

Dans la zone d'établissement du régime thermique, le coefficient de convection a une valeur plus élevée que celle donnée par la formule (6); on trouvera ci-dessous le résultal du calcul ri-

\begin{tabular}{|c|c|c|c|c|c|c|c|}
\hline$x / d \mathscr{R} \mathscr{T}$ & 0,001 & 0,002 & 0,005 & 0,01 & 0,02 & 0,04 & $0,05 \rightarrow$ \\
\hline $\mathcal{B}$ & 10,6 & 8,4 & 6,2 & 4,9 & 4,2 & 3,8 & $3,66 \rightarrow$ \\
\hline
\end{tabular}
goureux :

Nous insistons à nouveau sur le fait que les résultals précédents ne sont valables que si le régime dynamique est établi; on ne possède ancune donnée sur le, cas où les régimes dynamique et thermique s'établissent simultanément.

Il nous parait utile d'ailleurs de revenir sur cette question. L'établissement du régime dynamique nécessite un parcours $l / d=0,06 \alpha$; celui du régime thermique $l^{\prime} / d=0,05 \propto \mathcal{R}$. Si l'on a affaire à un gaz, pour lequel $\mathscr{T}$ est voisin de 1 , les régimes dynamique et thermique s'établissent après des parcours très voisins; il n'en est plus de même pour une huile dont le nombre de Prandtl peut dépasser 1.000: la longneur d'étitblissement $d u$ régime thermique est alors 1.000 fois plus grande que celle du régime dynamique. Pour un Reynolds égal à 200, le régime dynamique est établi au bout de 12 diamètres; le régime thermique nécessite 12.000 diamètres. Pratiquement, dans les réchauffeurs d'huile le régime thermique n'est jamais établi et l'adoption, pour $x$, de la formule (6) conduirait à des valeurs nettement trop faibles, comme l'indique le tableau précédent.

\section{Ecoulement turbulent dans un conduit}

$\mathrm{Si}$, dans un conduit, le nombre de Reynolds est supérieur à une valeur critique voisine de 2.000 , l'écoulement cesse d'être laminaire. Comme dans le cas du plan, la couche limite laminaire qui s'amorce à l'entrée tend à devenir turbulente et, après un parcours suffisant, la zone turbulente gagne l'axe du conduit (fig. 5). Le parcours $l$ né-

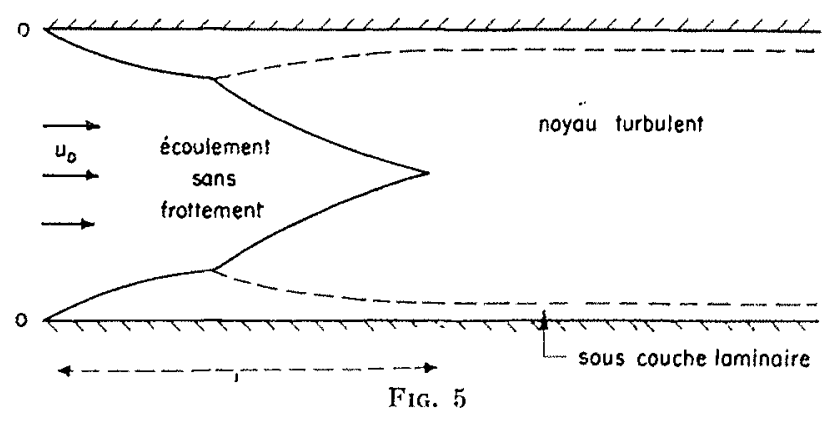

cessaire à l'établissemient du régime dynamique a pour expression :

$$
\frac{l}{d}=0,8 \mathfrak{R}^{1 / 4}
$$


Poux un Reynolds égal à 10.000 , ce parcours est égal à 8 diamètres.

Nous n'envisagerons pour l'instant que la zone de régime dynamique établi. La courbe des vitesses dans une section droite a l'aspect donné par la figure 6. Au voisinage immédiat de la paroi, la vitesse varie linéairement dans la sous-couche laminaire; dans la couche suivante appelée zone de transition, la viscosité habituelle et la turbulence se superposent; enfin dans la zone centrale on a une turbulence pure pour laquelle la loi de vitesses peut s'écrire approximativement: $u / u_{0}=(y / r)^{1 / \tau}$.

Dans la zone turbulente, le calcul de la convection est extrêmement facile. Je vous en donne les éléments essentiels basés sur le raisonnement suivant. Dans un écoulement turbulent, en un point nous avons une certaine vitesse d'ensemble que j'appellerai $u$, mais en ce point existent des fluctuations de vitesse, c'est-à-dire des vitesses désordonnées qui n'obéissent à aucune loi, qui sont purement accidentelles et que l'on peut, en réalité, représenter par une vitesse fluctuante $\boldsymbol{u}^{\prime}$ dirigée dans le sens de l'écoulement et une vitesse également aléatoire $v^{\prime}$ dirigée dans le sens perpendiculaire au mouvement d'ensemble. La vitesse $v^{\prime}$, transportant de la matière d'une tranche $y$ où la vitesse est $u$ et la température $T$ à la tranche voisine $y+d y$, où la vitesse et la température ont respectivement pour valeurs $u+d u$ et $\mathrm{T}+d \mathrm{~T}$, transporte dans cette tranche à la fois de la quantité de mouvement, proportionnelle à la masse $m$ transportée et à $d u$, et de la chaleur, proportionnelle à $m \mathrm{C}$ et à $d \mathrm{~T}$, de sorte que si l'on écrit le frottement unitaire local $f$ par la relation $f=u^{\prime}(d u / d y)$, la quantité de chaleur $Q$ traversant l'unité de surface s'écrira $Q=\mu^{\prime} \mathrm{C}(d \mathrm{~T} / d y)$ (raisonnement d'analogie de Reynolds), d'où la relation suivante:

$$
\frac{d \mathrm{~T} / d y}{d u / d y}=\frac{\mathrm{Q}}{f \mathrm{C}}
$$

Si l'on néglige la sous-couche laminaire et si $\mathrm{T}_{0}$ et $u_{0}$ sont la température et la vitesse sur l'axe, on a $\mathrm{T}_{0} / u_{0}=\hat{Q} / f \mathrm{C}$ et le coefficient de convection s'écrit :

$\alpha_{0}=\frac{\mathrm{Q}}{\mathrm{T}_{0}}=\frac{f \mathrm{C}}{u_{0}}($ formule de Reynolds-Stanton $) ;$

ou, en explicitant le coefficient de frottement :

$$
\begin{gathered}
\mathrm{C}_{f}=\frac{f}{(1 / 2) \bullet u_{0}{ }^{2}} \\
\mathfrak{K}_{11}=\frac{\alpha_{0}}{\mathrm{C}_{0} u_{10}}=\frac{\mathrm{C}_{f}}{2}
\end{gathered}
$$

relation qui met en relief le lien intime entre le frottement et la convection.

Dans le calcul précédent, nous n'avons pas tenu compte de la sous-couche laminaire et de la zone de transition. Pour les nombres de Prandtl supérieurs à l'unité, le calcul montre que les formules (8) et (9) doivent être remplacées par les suivantes :

$$
\begin{aligned}
\varkappa_{0} & =\frac{f \mathrm{C} / u_{0}}{1+\left(u^{\prime \prime} / u_{0}\right)^{\prime}\left(\mathscr{S}^{2 / 3}-1\right)} \\
\mathfrak{C}_{0} & =\frac{1}{1+\left(u^{\prime \prime} / u_{0}\right)\left(\mathscr{S}^{2 / 3}-1\right)}
\end{aligned}
$$

$u^{\prime \prime}$ représentant la vitesse au milieu de la zonc de transition.

Pour des nombres de Prandtl voisins de l'unité (cas des gaz), la formule ( 8 bis) se confond avec (8); pour des nombres de Prandtl élevés (huiles), le lerme en $\mathscr{T}$ au dénominateur prend une valeur prépondérante. Quant à la courbe des températures, pour les gaz elle se confond pratiquement avec la courbe des vitesses, pour les liquides elle se trouve de plus en plus au-dessus de la courbe des vitesses (fig. 6) et, pour une

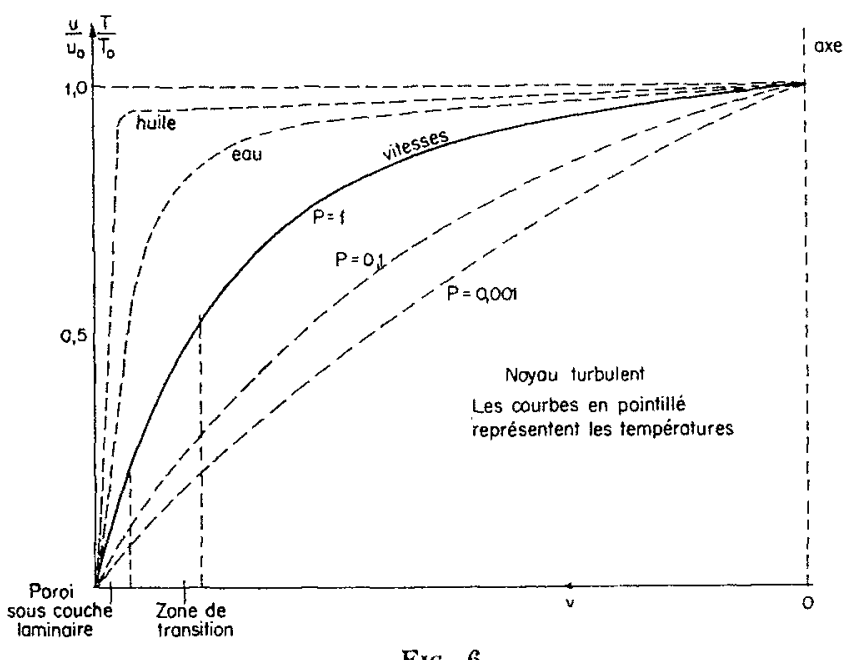

FxG. 6

huile, la chute de température s'effectue presque uniquement dans l'épaisseur de la sous-couche laminaire. Les métaux fondus, dont le nombre de Prandtl est nettement inférieur à l'unité, ont au contraire une courbe de température tout entière au-dessous de celle des vitesses; $\dot{a}$ la limite pour $\mathscr{P}_{r}=0$, elle tend vers une parabole et la chute de température dans la zone de transition reste faible par rapport à celle du noyau turbulent; le calcul conduit, dans ce cas, à une expression différente de 8 bis.

Bien entendu, les formules précédentes ne sont applicables que si les régimes dynamique et thermique sont établis; nous reviendrons sur la question dans un instant. 


\section{Cas du plan en régime turbulent}

Nous avons vu (fig. 2) qu'après un parcours suffisant le long d'un plan, l'écoulement devient turbulent. La répartition des vitesses prend alors le même aspect que dans le cas du conduit (fig. 6) et l'on est amené à adopter une formule inalogue à $(9$ bis $)$ et qui s'écrit :

$$
\frac{\alpha_{x}}{\mathrm{C} \rho u_{0}}=\frac{0,029 \mathcal{R}_{1}^{-0.20}}{1+\left(u^{\prime \prime} / u_{0}\right)\left(\mathscr{Q}^{2 / 3}-1\right)}(9 \text { ler })
$$

En pratique, cette formule conduit à un coefficient de convection moyen $\alpha_{m}$ qui, avec une approximation suffisante, peut s'écrire :

$$
\frac{\alpha_{m}}{C_{\rho} \rho u_{0}}=\frac{0,0017}{1+\left(u^{\prime \prime} / u_{0}\right)\left(\Phi^{2 / \hat{n}}-1\right)}
$$

Le dénominateur des diverses expressions précédentes, qui fait intervenir $\mathscr{T}$, montre que, toutes choses égales, si l'on passe d'un gaz $(\mathfrak{T}=1)$, à l'eau $(\mathscr{T}=8)$ et à une huile $(\mathscr{T}=125)$, ce dénominateur passe de 1 à 3 et à 18 .

\section{Cas où les régimes dynamique et thermique ne sont pas établis dans un conduit}

Nous avons fait remarquer plus haut que les formules relatives au conduit supposent les régime dynamique et thermique préalablement étahlis. Le problème de la convection à partir de l'entrée d'un conduit est fort compliqué en raison du fait que les parcours nécessaires à l'établissement des deux régimes peuvent être très différents, en particulier, comme nous l'avons vu, pour les liquides visqueux.

Un calcul approché ne peut être effectué que dans le cas des gaz où ces deux parcours $l$ sont les mêmes et tels que $l / d=0,08 \mathfrak{R}^{0.25}$.

I'n tel calcul revient, en fait, à traiter la partie d'entrée du conduit comme étant assimilable au cas du plan; le coefficient de convection, théoriquement infini au bord d'attaque, décroît pour tendre vers la valeur du régime au bout du parcours l. Pour un parcours égal à $l / 8$, le coefficient moyen est $40 \%$ plus élevé que le coefficient limite; pour de très faibles parcours, comme ceux qui se présentent dans les « convecteurs » d'auto ou d'avion, ce coefficient moyen peut devenir supérieur à trois fois le coefficient limite.

\section{Obstacles normaux au courant fluide}

Le cas le plus classique est celui d'un cylindre circulaire de diamètre $d$ dont l'axe est normal à la vitesse $u_{0}$ du fluide à l'infini.

Si le nombre de Reynolds $\alpha=u_{0} d /$ y est in- féricur à 4 , il se forme, à partir du point d'arrêt $A$ et jusquau point de fuite $F$ (fig. $7 a$ ) une conche limite laminaire et l'on a une assez bonne approximation en appliquant à cette couche la formule du plan, ce qui conduit à l'expression :

$$
\frac{\alpha_{n} d}{\lambda}=0,53 \mathcal{R}^{1 / 2} \mathscr{P}^{1 / 3}
$$
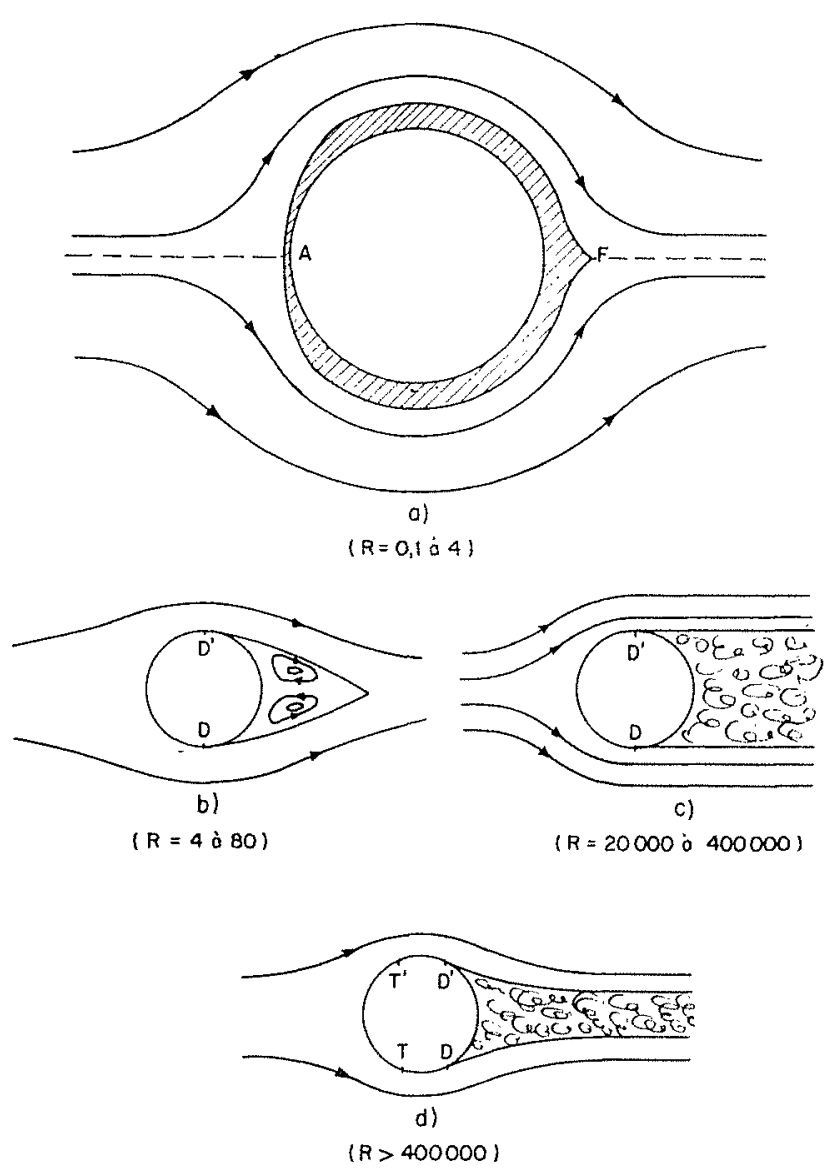

FiG. 7

Ce mode de calcul est d'ailleurs valable pour tout corps profilé dans lequel il n'y a fas décollement de la couche limite.

Pour des nombres de Reynolds supéricurs a 4 , il se produit un décollement de la couche limite avec, à l'arrière du cylindre, un sillage dont l'aspect varie avec $\mathcal{R}$; sur les figures $7 \mathrm{~b}$ et $7 \mathrm{c}$, l'écoulement avant reste laminaire jusqu'au point de décollement $D$ de la couche limite; sur la figure $7 d$, l'écoulement cesse d'être laminairc à partir du point de transition $\mathrm{T}$ et le décollement se produit en $D$.

Les calculs, donnés plus haut, restent valables jusqu'au point de décollement mais cessent de l'être dans le sillage.

La seule ressource est de faire appel au nombreux bagage expérimental obtenu avec des diamètres allant de quelques microns à plusieurs dizaines de centimètres. Au voisinage d'un Rey- 
nolds déterminé on peut exprimer le nombre de Biot-Nusselt par une formule de la forme:

$$
\mathfrak{B}_{m}=\frac{\alpha_{m} d}{\lambda}=\mathrm{A} \mathfrak{R}^{n} \mathscr{P}^{1 / 3}
$$

mais le nombre $A$ et l'exposant $n$ varient avec $\mathcal{R}: n$ varie de 0,33 à 0,80 lorsque $\mathcal{R}$ varie de 0,1 à 400.000 ; pour obtenir une représentation valable pour tous les fluides allant des gaz aux

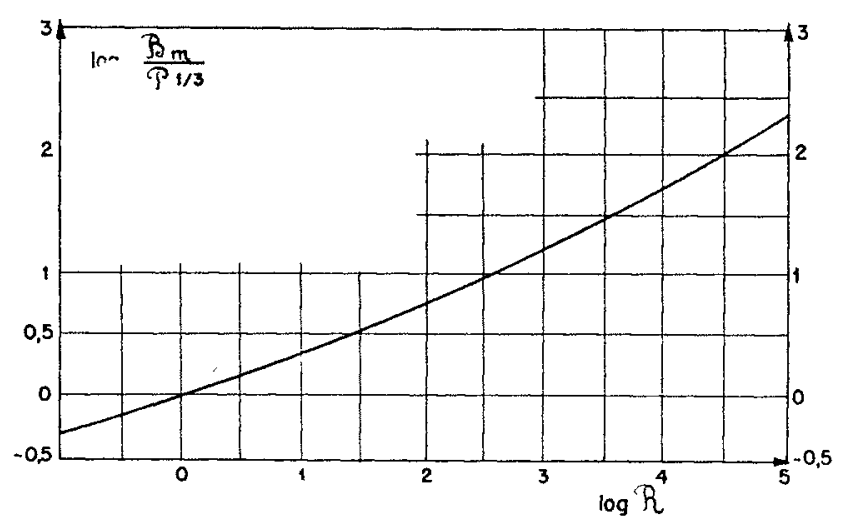

FIG. 8 huiles, il est infinimenl préférable de tracer la courbe qui donne $\log \mathscr{B}_{m} / \mathscr{P}^{1 / 3}$ en fonction de $\log \mathcal{R}$ (fig. 8). On voit que la pente de cette courbe, c'est-à-dire l'exposant $n$, varie de facon continue avec $\mathcal{R}$.

Je m'excuse d'avoir élé un peu long et d'avoir dépassé l'horaire prévu, mais j'ai droit à quelques excuses de votre part; étant donné l'ampleur du sujet, je n'ai pu que l'effleurer et vous en apporter les éléments essentiels.

Ce sur quoi je voudrais revenir, c'est sur le fait que lorsqu'on n'est pas averti des problemes de convection et que l'on cherche dans les formulaires ou dans les ouvrages techniques, on trouve des formules très simples; mais le danger est que trois fois sur quatre, pour ne pas dire neuf fois sur dix, ces formules ne sont pas rigoureusement applicables parce que le régime thermique ou le régime dynamique ne sont pas établis.

Ceci revient à dire que le thermicien, appelé à résoudre des problèmes de convection, doit commencer par faire de la mécanique des fluides s'il vent éviter de commettre de grossic̀res erreurs.

\section{DIS CUSSION}

président : M. Baumgartner

M. le Président remercic $M$. le Professeur Ruaud et ouvre la discussion.

Après avoir signalé un lapsus qui s'était glissé dans l'exposé, M. AcLogue, considérant que, pendant les périodes de régime transitoire, on bénéficie, aussi bien en régime laminaire qu'en régime turbulent, d'une augmentation considérable du coefficient de transfert de chaleur, demande si l'on pourrait concevoir un régime d'écoulement pulsé dans lequel les périodes transitoires seraient seules existantes ou, tout au moins constitucraient l'essentiel du régime.

$M$. Ribaud répond que, dans le cas général, si on veut augmenter la convection, on augmente parallèlement le frottement. C'est valable pour des échangeurs comme pour des écoulements dans des conduites, ou le long de plans, ou dans des faisceaux d'ailettes, par exemple. On peut effectivement gagner beaucoup an point de vue convection en mettant des obstacles sur le parcours, mais c'est au détriment de la perte de charge et, comme cette perte de charge dans beaucoup de cas joue un rôle essentiel, en pratique on est obligé de chercher un compromis. 